\title{
ANÁLISE DO BEM ESTAR PSICOSSOCIAL DE APOSENTADOS DE GOIÂNIA
}

\author{
Catarino de Oliveira* \\ Ana Raquel Rosas Torres \\ Eduardo Simões de Albuquerque
}

\begin{abstract}
RESUMO. Este estudo analisou o bem estar psicossocial de aposentados da cidade de Goiânia. Participaram 118 homens, aposentados por tempo de serviço, cuja vida laboral ocorreu em empresas públicas, autarquias e de economia mista, de âmbito federal, estadual ou municipal. A coleta de dados foi realizada por meio de questionário com perguntas abertas e fechadas. Para a análise dos resultados foi realizada uma regressão múltipla, tendo como variável critério o índice de bem estar e, como seus possíveis preditores, oito variáveis sobre aspectos referentes aos relacionamentos sociais, vida financeira, avaliação da saúde e da vida sexual após a aposentadoria. As variáveis sobre o planejamento, satisfação com a saúde e as relações sociais nas quais eles possam se locomover com independência foram as mais significativas para o bem estar dos participantes. Esses resultados são discutidos ressaltando as especificidades da amostra aqui utilizada, as dificuldades de generalizações e a importância de futuros estudos objetivando tornar esse tema central na agenda de trabalhos sobre o envelhecimento populacional.
\end{abstract}

Palavras-chave: Aposentados; bem estar psicossocial; aposentadoria.

\section{ANALYSIS OF RETIRED MEN'S PSYCHOSOCIAL WELL-BEING IN GOIÂNIA}

\begin{abstract}
This study examined the psychosocial well-being of retired on pension men in Goiânia. The participants were 118 men, retired by service time, whose working life occurred in public service companies. The instrument used was a questionnaire with open and closed questions. Multiple regressions were used to analyze the data. The dependent variable was the well being index and the predictors were eight variables on aspects related to social relationships, financial life, health and sexual life after retirement. The most significant predictors for the well being of the participant were living the retirement as planned, satisfaction with their health and the possibility of social relationships where they can move more independently. These results are discussed emphasizing the peculiarities of the sample, the difficulties to generalize the results and the importance of further studies aiming at making the subject central to agenda of studies on population aging.
\end{abstract}

Key words: Retired; psychosocial well-being; retirement.

\section{ANÁLISIS DEL BIEN ESTAR PSICOSOCIAL DE JUBILADOS DE GOIÂNIA}

RESUMEN. Este estudio analizó el bienestar psicosocial en una muestra de jubilados de la ciudad de Goiânia. Participaron 118 hombres, jubilados tras finalizar su vida laboral, cuyo trabajo transcurrió en empresas públicas y de economía mixta, de ámbito nacional, comarcal o municipal. La recogida de datos fue realizada por medio de la aplicación de un cuestionario con preguntas abiertas y cerradas. Para la obtención de los resultados fue realizado un análisis de regresión múltiple, en el que la variable criterio fue el índice de bienestar y como variables predictoras se incluyeron ocho variables acerca de aspectos referidos a las relaciones sociales, situación económica, evaluación de la salud y de la vida sexual después de la jubilación. Las variables eferidas a la planificación, la satisfacción con la vida y las relaciones sociales que permiten desplazarse con independencia fueron las más significativas en la explicación del bienestar de los participantes. En la discusión de los

1 Este trabalho é parte da dissertação defendida pelo primeiro autor intitulada "Análise do bem estar psicossocial de aposentados do município de Goiânia".

* Mestre, Universidade Católica de Goiás, Goiânia-GO, Brasil.

\# Doutora em Psicologia. Professora do Programa de Pós-Graduação em Psicologia, Universidade Católica de Goiás, Goiânia-GO, Brasil.

Il Doutor em Ciência da Computação. Professor do Programa de Pós-Graduação em Ciências Ambientais e da Saúde, Universidade Católica de Goiás, Goiânia-GO, Brasil. 
resultados obtenidos se destaca la especificidad de la muestra utilizada, las dificulatades para poder generalizar los resultados y la necesidad de realizar futuras investigaciones que tengan como objetivo el estudio del envejecimiento poblacional.

Palabras clave: Jubilados; bienestar psicosocial; jubilación

A expectativa de vida dos brasileiros, entre $1991 \mathrm{e}$ 2007, aumentou 5,57 anos, passando de 67 anos para 72,57 anos (IBGE, 2009). Em Goiás, essa expectativa é de 73,4 anos, sendo essa taxa a nona maior do Brasil. $\mathrm{O}$ envelhecimento da população tem diversas consequências, como por exemplo, o aumento de doenças degenerativas próprias da velhice e a sobrecarga do sistema previdenciário, uma vez que as pessoas recebem a aposentadoria por um período maior de tempo (Izerrougene, 2009).

A aposentadoria é um fenômeno historicamente recente no Brasil, pois o primeiro instituto de aposentadoria e pensões foi criado em 1934. A possibilidade de planejamento da aposentadoria está diretamente ligada às condições governamentais. As políticas previdenciárias, econômicas, de saúde e de educação proporcionam ou não melhorias para a execução e o entendimento acerca do processo de aposentadoria.

A aposentadoria revela uma ambivalência, pois ao mesmo tempo em que é a conquista, por meio do trabalho, do tempo livre, é também marginalizada, como uma inutilidade, pela sociedade produtiva, e como um período de decadência, pela concepção social que valoriza o belo, o forte, o jovem e o saudável. A aposentadoria deveria ser o auge do bem estar psicossocial, pois, desde o nascimento, o homem prepara-se para trabalhar e, no futuro aposentar-se. Entretanto, ante as características de subjetividade e construção sócio-histórica de cada um, as alternâncias nas políticas governamentais e as constantes transformações ambientais, esse fenômeno social requer permanentes pesquisas visando compreender a qualidade das aposentadorias e o bem estar psicossocial dos ex-trabalhadores. Diante deste contexto, este trabalho objetivou analisar o bem estar psicossocial de trabalhadores aposentados da cidade de Goiânia.

Kalache, Veras e Ramos (1987) e Izerrougene (2009) alertam para o rápido crescimento previsto para a população idosa em países como o Brasil nas próximas décadas, indicando a necessidade de estimular o estudo da terceira idade no contexto do terceiro mundo. A pequena ênfase dada a esse tema pode ser ilustrada pelo número limitado de investigações relativas às implicações sociais, econômicas e ambientais causadas por mudanças na estrutura etária como as que a população brasileira atravessa.
Uma pesquisa feita no site da Revista de Psicologia Organizacional e Trabalho (http://www.periodicos.ufsc.br/index.php/rpot) no dia 19 de setembro de 2009, usando "aposentadoria" e "envelhecimento" como palavras de busca não resultou em nenhum artigo publicado. $\mathrm{O}$ mesmo procedimento foi feito no site de pesquisa bibliográfica scielo (www.scielo.br) usando "aposentadoria". Os resultados mostraram apenas 13 artigos publicados, dos quais quatro se referem aos aspectos econômicos, dois falam de aposentadoria por invalidez e sete investigam os aspectos psicossociais e da saúde envolvidos na transição para a aposentadoria. Esses sete trabalhos são discutidos mais à frente.

No entanto, o momento da aposentadoria apresenta, geralmente, notável relevância para os sentimentos e valores, quando também surgem temores ligados ao pressentimento de inutilidade, de isolamento, de dificuldades financeiras e de desatualização. Trata-se de um processo subjetivo que claramente envolve crenças e emoções, com a percepção de mundo de cada um. Assim, a préaposentadoria é um momento propício para a reflexão e análise de questões pertinentes à própria identidade, às expectativas e prioridades para o futuro. Zanelli (2000), por exemplo, analisou os programas preparatórios para a aposentadoria e constatou sua importância durante a passagem da vida laboral para a aposentadoria.

Com base em entrevistas realizadas com 51 canadenses aposentados, com idade entre 45 e 91 anos, Kemp, Rosenthal e Denton (2005) assinalaram que as circunstâncias que envolvem finanças e relacionamentos pessoais e familiares podem atuar tanto como pontos que facilitam ou dificultam o planejamento da aposentadoria. Para o trabalho ora aqui apresentado, é importante destacar, conforme a pesquisa de Kemp et al. (2005), que 80\% dos entrevistados identificaram a preocupação com a condição financeira como um fator motivador para a construção de uma poupança para a aposentadoria.

Investigando 2.886 idosos do sexo masculino, com 65 anos ou mais de idade, residentes em regiões metropolitanas brasileiras e participantes da Pesquisa Nacional por Amostra de Domicílios de 1998, Giatti e Barreto (2003) constataram que $68,46 \%$ dos entrevistados eram aposentados, e, entre os idosos que ainda trabalhavam, $64 \%$ estavam na informalidade, ou seja, não contribuíam para a previdência social. Os ramos de atividade econômica que concentravam as 
maiores proporções de idosos trabalhando foram os de prestação de serviço e comércio. Os resultados desse trabalho também demonstraram que um pouco mais da metade $(57,83 \%)$ dos aposentados não tinham plano de previdência privada.

A importância do tipo da profissão na composição do bem estar na aposentadoria foi demonstrada na pesquisa de Blekesaune e Solem (2005) com 1.990 aposentados noruegueses, com idade entre 60 e 67 anos. Esse estudo evidenciou que trabalhos estressantes podem levar à aposentadoria precoce, mas, sobretudo, que os homens com baixa autonomia nos trabalhos se aposentam mais cedo do que aqueles que possuem maior flexibilidade de decisão sobre suas atividades laborais. A explicação dada por esses autores para os resultados foi que a flexibilidade estaria ligada à satisfação com a tarefa desempenhada, que, para os participantes, poderia fazer falta na aposentadoria, daí porque o adiamento.

Entrevistando funcionários públicos estaduais aposentados há mais de cinco anos, Campos e Chamon (2002) verificaram que o funcionário público, detentor da estabilidade no emprego, tem um discurso que apresenta frequentemente uma valorização positiva em relação ao trabalho. Para os autores, essa tendência do julgamento positivo da profissão pode ser entendida com base na ancoragem em realidades preexistentes de valorização da função pública e na rede de representações construída, proporcionadas pela estabilidade, pela aposentadoria integral e status inerente ao cargo.

Analisando os discursos de treze aposentados da cidade de Belém (PA), Torres (2002) apurou que o trabalho nas sociedades modernas é o grande indicativo do valor do indivíduo, e que, ao aposentarse, o indivíduo tem uma sensação de vazio, e um desconsolo instala-se ao interromper aquilo que considerava o grande objetivo de sua vida. A interrupção compulsória do trabalho, apoiada no referencial cronológico, ignora as capacidades reais físicas e mentais das pessoas para desempenhar atividades produtivas. O valor social do trabalho, a segurança e estabilidade transformam-se rapidamente em passado, como o exemplo de um militar, que passou de ativo para inativo ou da reserva.

Torres (2002) apurou também que os aposentados entrevistados consideram o trabalho uma inserção obrigatória no sistema de relações econômicas e sociais, que penetra no seu dia-a-dia e se confunde com o próprio sentido da vida. Na sociedade moderna, a idade burocrática para decidir até quanto uma pessoa pode trabalhar é utilizada como uma das demarcações para definir a velhice. $\mathrm{Na}$ contemporaneidade, a experiência não é mais trocada entre gerações, e as conseqüências físicas da velhice não incomodam tanto quanto o afastamento do mundo do trabalho, a dispersão da família, as modificações nas relações com os amigos e na sociedade e a perspectiva da morte.

Os resultados encontrados por Pinquart e Schindler (2007) parecem apoiar essas idéias. A partir de entrevistas realizadas com 1.456 aposentados alemães esses autores mostram que, nos primeiros anos de aposentadoria existe um ligeiro aumento da satisfação com a vida. No entanto, na medida em que os anos vão passando, começa a existir um declínio no bem estar dessas pessoas. Esse declínio pode ser explicado por dois fatores: ao aumento das limitações físicas, próprias do envelhecimento, mas também pela ausência dos vínculos sociais propiciados pela atividade laboral.

Mais recentemente, França (2009) analisou a influência dos preditores sociais nas atitudes em face da aposentadoria em 517 altos executivos do Brasil e da Nova Zelândia. Os resultados indicam que quanto mais positiva for a influência da família e dos amigos, maior será a importância dos ganhos atribuídos na aposentadoria para ambas as nacionalidades. Essa importância também é aumentada pela diversidade na alocação de tempo entre as atividades/relacionamentos, mas apenas para os executivos brasileiros. Os executivos brasileiros que percebem, positivamente, seus trabalhos, demonstram atitudes mais positivas nos relacionamentos, no lazer, nos hobbies e atividades culturais na aposentadoria. Essa autora (França, 2008) também demonstrou, junto aos mesmos participantes, que os executivos dos dois países estavam otimistas acerca da aposentadoria e foram unânimes em avaliar o tempo para os relacionamentos (cônjuge, filhos, amigos e pais) como o ganho mais importante, bem como os salários e benefícios do cargo, como a perda mais importante nesta transição.

Pimenta et al. (2008) desenvolveram um estudo cujo objetivo era investigar os fatores que poderiam influenciar positivamente a qualidade de vida de idosos. Para tanto, eles entrevistaram 87 aposentados residentes em Belo Horizonte. Os resultados apontaram a percepção de uma melhor qualidade de vida nos aposentados que praticavam atividade física regular ou que tinham alguma atividade de trabalho no momento da pesquisa, ou seja, que mantinham algum tipo de atividade que favorece o estabelecimento de relações interpessoais.

Buscando identificar fatores associados ao envelhecimento bem-sucedido, Moraes e Souza (2005) investigaram quatrocentos idosos, com idade média de 67 anos, da região metropolitana de Porto Alegre (RS), empregando a escala de Flanagan e o instrumento de qualidade de vida WHOQOL-100, da 
Organização Mundial de Saúde. Esses autores constataram que a manutenção da independência para as atividades da vida diária, autonomia e satisfação com relacionamentos familiares e amizades foram fatores preditivos do envelhecimento bem-sucedido, tanto para homens como para mulheres.

Santos et al. (2002) avaliaram a satisfação de 128 idosos, com 60 anos ou mais, em relação a sua qualidade de vida utilizando a escala de qualidade de vida de Flanagan (EQVF). Eles constataram que os resultados em alguns itens divergem daquela escala, sugerindo que em relação à EQVF pode haver variações na ordem de prioridades das dimensões (bem estar físico e material, relações com outras pessoas, atividades sociais e comunitárias, desenvolvimento pessoal e realização, e recreação), dependendo da população estudada e do contexto dos participantes. A pesquisa demonstrou que a qualidade de vida dos idosos vai de pouca a moderada satisfação, resultante, segundo os autores, da condição biopsicossocial experimentada no contexto brasileiro, sobretudo em razão das tribulações e agressões econômicas, sociais e ambientais.

Utilizando a meta-análise da revisão sistemática de 286 pesquisas que envolviam participantes com idade entre 55 e 60 anos, Pinquart e Sorensen (2000) evidenciaram que pessoas idosas com alto status sócio-econômico, com melhor relacionamento social e com independência e nível de habilidades que facilitam a relação com o meio-ambiente, têm satisfação com a vida e auto-estima mais elevadas. A influência do status sócio-econômico no bem estar subjetivo é maior no homem do que na mulher. No caso das mulheres, a influência de uma rede de apoio social, que privilegie as relações interpessoais, é maior.

O bem estar subjetivo foi definido por Pinquart e Sorensen (2000) como a avaliação positiva da vida de uma pessoa, associada com os sentimentos bons, e envolvendo a boa relação com diversos aspectos da vida, como o status sócio-econômico e uma rede de apoio social. Os autores constataram em sua metaanálise que, ao contrário do que é sugerido, o bem estar de pessoas idosas não é mais baixo do que em pessoas mais novas, podendo ser avaliado qualitativamente por meio da auto-estima, satisfação com a vida e felicidade. Nas diferenças entre gênero, as mulheres são mais afetadas positivamente pelos aspectos que envolvem relações familiares do que os homens. Nas amostras masculinas estudadas, foi constatado uma influência positiva mais elevada de status sócio-econômico no bem estar do que nas amostras femininas. $\mathrm{Na}$ rede social, os relacionamentos com amigos podem ser mais importantes para o bem estar subjetivo do que a relação com a família, porque os amigos são mais voluntários e flexíveis e compartilham o mesmo grupo de idade e têm estilo de vida semelhante.

Em outra meta-análise de setenta pesquisas que envolviam participantes com idade abaixo de quarenta anos ou acima de sessenta anos, Pinquart (2002) focou no objetivo de vida, estabelecendo associações entre saúde física, competências, bem estar subjetivo, status sócio-econômico e depressão. Nesse trabalho, o autor identificou que as relações sociais têm grande relevância na associação com os propósitos de vida, evidenciando ser um fator motivador para atividades de colaboração com outras pessoas, como forma de promover um sentimento de utilidade e respeito. Também de grande importância para este estudo é a constatação de que os relacionamentos sociais interferem no bem estar psicológico dos aposentados.

$\mathrm{O}$ bem estar subjetivo da pessoa mais velha pode ser tão elevado quanto o de pessoas mais novas, com exceção de pessoas aposentadas por problemas de saúde, em vista dos efeitos psicológicos negativos, que geram nessas pessoas uma satisfação com a vida menor do que os aposentados por tempo de serviço. $\mathrm{O}$ bem estar pode estar ligado à posição durante o emprego, e entre as pessoas empregadas e aposentadas não há diferenças significativas, mas ambos os grupos possuem um bem estar mais elevado que os desempregados. Entretanto, os indivíduos empregados e com idade de aposentadoria têm um bem estar afetivo mais elevado do que os já aposentados (Warr, Butcher, Robertson \& Callinan, 2004).

Estudando os discursos de 365 indivíduos com sessenta anos ou mais, com base no que é qualidade de vida, Vecchia et al. (2005) identificaram três perfis de idosos: a) o idoso que prioriza a questão afetiva e a família; b) o idoso que prioriza o prazer e o conforto; c) o idoso que identifica como qualidade de vida conseguir colocar em prática o seu ideário de vida. Para este trabalho, é importante destacar que o maior grupo é o dos idosos que priorizam o afeto e a família como aspectos importantes na qualidade de vida, reforçando que as investigações sobre o bem estar dos aposentados devem conter questões sobre o apoio social.

Analisando os estudos que tratam da qualidade de vida dos idosos, relacionada às maneiras como os indivíduos enfrentam suas perdas em funções físicas e papéis sociais e a extensão em que os recursos psicológicos e sociais operam sobre os efeitos negativos das condições crônicas, Rabelo e Néri (2005) concluíram que as definições e indicativos de qualidade de vida podem variar desde o status sócio- econômico, a satisfação de necessidades e a capacidade funcional até o sentido da vida, a satisfação com a vida, o bem estar e a felicidade. Para os indivíduos com incapacidades, a boa qualidade de vida depende do balanço entre corpo e 
mente e da manutenção de relações harmoniosas nos seus contextos pessoais e sociais.

Utilizando uma amostra randômica de $35 \%$ dos idosos com mais de oitenta anos residentes em Veranópolis (RS), Xavier, Ferraz, Marc, Escosteguy e Moriguchi (2003) procuraram identificar a prevalência de uma avaliação da vida atual em que a qualidade fosse positiva e os domínios que foram determinantes para essa qualidade positiva. A aplicação do questionário semi-estruturado de qualidade de vida resultou que, comparados com os satisfeitos, os insatisfeitos tinham mais problemas de saúde e mais sintomas depressivos. Os insatisfeitos declararam sobretudo a falta de saúde física. Ainda para Xavier et al. (2003), os determinantes da boa qualidade de vida na velhice variam de sujeito para sujeito. Entretanto, nos seus estudos, o aspecto saúde parece um bom indicador de qualidade de vida positiva.

Pereira et al. (2006) avaliaram a contribuição dos domínios físico, social, psicológico e ambiental para a qualidade de vida global, definida como a satisfação geral do indivíduo com a vida e a percepção geral do bem estar, de 67 idosos, com idade igual ou superior a 60 anos. A maioria deles era de baixa renda e baixa escolaridade e eram participantes do Programa Saúde da Família de uma cidade interiorana. Os autores utilizaram o instrumento WHOQOL-brief-versão brasileira, da Organização Mundial de Saúde. Segundo os resultados, os idosos participantes apresentaram bons escores de qualidade de vida para todos os domínios do instrumento, sendo o domínio físico o que mais contribui na qualidade de vida global, seguido do ambiental e do psicológico. Somente a variável gênero teve influência significativa, porém pequena, nos domínios físico, psicológico e ambiental, sendo os escores médios desses domínios maiores entre os homens. Os domínios social e psicológico não se mostraram fortes influentes na qualidade de vida global, porque, segundo Pereira et al (2006), o grupo estudado passa pelas alterações psicossociais do envelhecimento de forma mais branda, pois nos trabalhos domésticos e rurais a perda de papéis funcionais pode não ocorrer com a aposentadoria, além de o ambiente rural facilitar a locomoção e convívio entre os indivíduos.

Diante da literatura aqui revisada, o objetivo geral deste trabalho foi analisar o bem estar psicossocial dos aposentados da cidade de Goiânia que trabalharam em empresas públicas, autarquias e de economia mista.

\section{MÉTODO}

\section{Participantes}

Os critérios de inclusão dos participantes foram: homens, aposentados por tempo de serviço, residentes em Goiânia, cuja vida laboral ocorreu em empresas públicas, autarquias e de economia mista, de âmbito federal, estadual ou municipal. A amostragem foi nãoprobabilística (Maroco, 2003), e teve como critério a acessibilidade aos possíveis participantes que preenchiam esses critérios.

\section{Instrumento}

O instrumento utilizado foi um questionário composto por questões abertas e fechadas, distribuídas em três seções. Na primeira, foram perguntados os dados sóciodemográficos, como: idade, estado civil, escolaridade, tempo de aposentadoria, rendas, última função ocupada antes de aposentar, se tem planos de saúde e aposentadoria, se tem carro próprio, quantas horas trabalhava antes de aposentar, onde morava antes de Goiânia e se participou de algum programa preparatório para a aposentadoria. Na segunda seção havia a escala do bem estar físico e psicológico, em formato likert, com 32 itens, variando de 1 (muito) a 7 (nem um pouco), que segue abaixo. Essa escala foi utilizada por Paula e Torres (2001), com motoristas de ônibus de Goiânia, e por Rabelo e Torres (2005), com trabalhadores em saúde mental. Nesses dois trabalhos, a análise fatorial mostrou a existência de um único fator, que foi transformado em um único índice que obteve níveis de validade considerados excelentes (Maroco, 2003), com o alfa de Cronbach variando entre 0,90 e 0,96 . Os itens dessa escala se encontram no final deste trabalho. Na terceira parte do questionário havia oito questões sobre a aposentadoria, também no formato Likert.

\section{Itens da Escala do Bem Estar}

1) Em geral, como você tem se sentido?

2) Você tem estado nervoso?

3) Você tem sentido que seus comportamentos, pensamentos e sentimentos estão sob controle?

4) Você tem se sentido triste, desencorajado, desesperançado ou tido muitos problemas que o tem preocupado?

5) Você tem estado sob tensão, estresse ou pressão?

6) O quanto você tem estado feliz, satisfeito ou contente com sua vida pessoal?

7) Você tem tido razão para pensar que está perdendo o controle sobre o seu modo de agir, falar, pensar ou sentir?

8) Você tem estado ansioso, preocupado ou indisposto?

9) Você tem acordado bem disposto e descansado?

10) Você tem sido perturbado por alguma doença, desordem corporal, dores ou medos sobre sua saúde?

11) Sua vida diária tem sido cheia de coisas que são interessantes para você?

12) Você tem se sentido deprimido ou magoado?

13) Você tem se sentido emocionalmente estável e seguro de si? 
14) Você tem se sentido cansado, esgotado ou exausto?

15) Você tem estado preocupado com sua saúde?

16) Quanta energia, dinamismo e vitalidade você tem sentido?

17) Você tem tido sérios problemas pessoais, emocionais, comportamentais ou mentais que o tenha feito sentir necessidade de ajuda?

18) Quanto você tem estado relaxado ou tenso?

19) Você tem estado deprimido ou alegre?

20) Você tem sentido que vai ter ou está perto de ter um esgotamento nervoso?

21) Você discute seus problemas com algum membro de sua família ou amigos?

22) Você tem conseguido se concentrar no que faz?

23) Você tem perdido o sono com preocupações?

24) Você se sente útil na vida?

25) Você se sente capaz de tomar decisões?

26) Você se sente constantemente sob pressão?

27) Você tem tido prazer em fazer suas atividades normais, do dia-a-dia?

28) Você tem a sensação de não poder superar as dificuldades?

29) Você tem se sentido capaz de enfrentar seus problemas?

30) Você tem pensado em si mesmo como uma pessoa sem valor?

31) Você tem perdido a confiança em si mesmo?

32) Você sente que está a beira de um esgotamento nervoso?

\section{Procedimentos}

O estudo foi realizado em seis associações de aposentados de Goiânia, contatadas previamente. O convite para a participação nesta pesquisa foi feito de forma individual e grupal, aproveitando eventos das associações, quando os participantes eram abordados. $\mathrm{Na}$ oportunidade, foi esclarecido ao aposentado como seriam os procedimentos e a forma de utilização dos resultados da pesquisa.

Após a assinatura do Termo de Consentimento Livre e Esclarecido (TCLE), foi entregue o instrumento de pesquisa e orientado sobre a devolução, feita por meio de depósito em uma urna específica que estava em cada associação de aposentados. O participante poderia responder ao questionário na própria associação de aposentados ou levá-lo para devolução posterior.

Os dados coletados foram analisados estatisticamente usando o SPSS (Statistical Package for Social Sciences) versão 11. Para analisar a estrutura fatorial da escala de bem estar, foi utilizada a Análise Fatorial, aliada ao teste de esferecidade de Bartlett e o teste de Kaiser-Meyer-Olkin que, juntos avaliam a adequabilidade da solução estrutural para os dados analisados (Maroco, 2003). Para investigar os possíveis antecedentes do bem estar dos participantes deste trabalho, foi utilizada a regressão múltipla.

\section{RESULTADOS E DISCUSSÃO}

A idade média dos participantes é de 64,7 anos, com Desvio Padrão (DP) igual a 6,8 anos e idade mínima de 50 e máxima de 79 anos.

A maioria dos participantes $(78,8 \%)$ é casado ou tem companheira, $38,3 \%$ possuem ensino médio e $46,1 \%$ nível superior. A maioria $(69,8 \%)$ possui plano de previdência complementar, $95,6 \%$ possuem plano de saúde e $94,7 \%$ possuem carro próprio. A média de tempo em que os participantes estão aposentados é de 12,1 anos com DP = 6,2 anos e tempo mínimo de um e máximo de 34 anos de aposentadoria, com renda média da aposentadoria de $\mathrm{R} \$ 4.859,88$, com $\mathrm{DP}=\mathrm{R} \$$ 3.037,41,

Ante essa nova situação - aposentado -, com maior horizonte temporal devido à longevidade, a pesquisa demonstra que ainda são reduzidas as ações preparatórias para a aposentadoria, durante a vida laboral, pois $89 \%$ dos respondentes não participaram de nenhum programa para tal fim.

Dos participantes, 69,2\% tomam algum remédio diariamente, e, destes, $50,0 \%$ possuem problemas de pressão, $15,9 \%$ diabetes e $13,4 \%$ colesterol.

\section{Análise da escala de bem estar}

Para a verificação do nível de bem estar foi calculada a análise fatorial, cujos resultados mostram a existência de um único fator com valor próprio igual a 15,3 e que explica $48 \%$ da variância dos itens da escala. O teste de esfericidade de Bartlett (3078,0 ; $\mathrm{p}<0,000)$ e o teste de Kaiser-Meyer-Olkin $(0,90)$ foram também significativos, indicando que realmente essa é a melhor solução estrutural para a escala. Finalmente, o alfa de Cronbach foi igual a 0,96, mostrando assim que a somatória dos itens da escala pode ser usada como uma única variável, denominada aqui como índice de bem estar. Importante ressaltar que esses resultados são semelhantes aos encontrados por Paula e Torres (2001) e Rabelo e Torres (2005). A média desse índice foi de 5,7 ( $\mathrm{DP}=1,1)$, indicando que os participantes possuem um índice de bem estar elevado, para uma escala do tipo Likert de sete pontos.

Em seguida, foi realizada uma regressão múltipla objetivando investigar quais os aspectos vivenciais da aposentadoria poderiam explicar o bem estar dos participantes deste estudo. Segundo Maroco (2003), a Análise de regressão é utilizada quando se quer predizer uma variável dependente contínua a partir de um conjunto de variáveis independentes. A análise de regressão não determina relacionamentos causais. 
Assim, com seu uso podemos dizer que $\mathrm{X}$ "prevê" $\mathrm{Y}$, mas não podemos afirmar que $X$ "causa" $Y$. Nesta análise utilizou-se o método seleção Stepwise. A vantagem deste método é que se pode remover uma variável cuja importância no modelo é reduzida pela adição de novas variáveis (Maroco, 2003).

Para o cálculo, foi utilizado como variável critério o índice de bem estar e como seus possíveis preditores as seguintes variáveis:

- Se vivem a aposentadoria conforme o planejado.

- Se a saúde melhorou depois da aposentadoria.

- Se avalia que fazer novas amizades ficou mais fácil após a aposentadoria.

- Se avalia que as relações familiares melhoraram após a aposentadoria.

- Se está satisfeito com a condição financeira.

- Se avalia a vida sexual como melhor.

- Se está satisfeito com o acesso aos serviços de saúde.

- Se está satisfeito com o acesso aos meios de transportes.

Os resultados da Tabela 1 mostram que apenas os itens "viver aposentadoria como planejou", "satisfeito com seu meio de transporte", "fazer amigos após aposentadoria" e "saúde melhor após aposentadoria" são significativos para o Bem estar. Cabe esclarecemos que, de acordo com a direção das escalas utilizadas para avaliar essas variáveis, são os fatores que melhor explicam o bem estar dos participantes. De fato, juntos, esses fatores explicam $43 \%$ da variância do bem estar, índice considerado excelente por Maroco (2003).

Tabela 1. Regressão Múltipla dos Preditores do Bem Estar Psicossocial

\begin{tabular}{lccc}
\hline & \multicolumn{3}{c}{ Bem estar } \\
\cline { 2 - 4 } & Beta & t & p \\
\hline $\begin{array}{l}\text { Vive aposentadoria como } \\
\text { planejou }\end{array}$ & $-0,365$ & $-4,076$ & $<0,00^{*}$ \\
$\begin{array}{l}\text { Satisfeito com seu meio de } \\
\text { transporte }\end{array}$ & $-0,176$ & $-2,045$ & $<0,01^{*}$ \\
$\begin{array}{l}\text { Fazer amigos após } \\
\text { aposentadoria }\end{array}$ & $-0,207$ & $-2,460$ & $<0,01^{*}$ \\
$\begin{array}{l}\text { Saúde melhor após } \\
\text { aposentadoria }\end{array}$ & $-0,203$ & $-2,174$ & $<0,032^{*}$ \\
$\begin{array}{l}\text { Satisfeito com acesso à saúde } \\
\text { Vida sexual melhor }\end{array}$ & $-0,148$ & $-1,837$ & $<0,765$ \\
$\begin{array}{l}\text { Relacionamentos familiares } \\
\text { melhores }\end{array}$ & 0,125 & 1,585 & $<0,839$ \\
$\begin{array}{l}\text { Dinheiro suficiente } \\
\text { Coeficiente de Regressão }\end{array}$ & 0,082 & $-1,899$ & $<0,789$ \\
$\begin{array}{l}\text { Variância Explicada } \\
\text { Significância do Modelo }\end{array}$ & $\mathrm{F}(8,106)=11,68$ & $\mathrm{R}<0,001$ & \\
\hline
\end{tabular}

Assim, para os participantes, quanto mais estão satisfeitos com todos esses aspectos da sua vida cotidiana, maior é seu bem estar. Aqui é importante ressaltar que, contrário ao esperado, as relações familiares e a condição financeira não foram significativas na predição do bem estar.

Destaque para a significância do planejamento da aposentadoria. Estudos anteriores de Ekerdt e Clark (2000) demonstraram um crescimento na preocupação dos trabalhadores em relação à aposentadoria, estando, entretanto, mais focado quando ela se aproxima, com forte influência no processo decisório as questões relacionadas aos incentivos financeiros e de saúde.

\section{CONSIDERAÇÕES FINAIS}

As análises estatísticas dos dados coletados apontam para uma tendência ao bem estar, sendo os itens "viver aposentadoria como planejou", "satisfeito com seu meio de transporte", "fazer amigos após aposentadoria" e "saúde melhor após aposentadoria" os mais significativos para o bem estar dos participantes da pesquisa.

Destaque para o fator planejamento na satisfação com os aspectos da vida após a aposentadoria. $\mathrm{O}$ planejamento é salutar em todas as áreas da vida, e na aposentadoria não seria diferente, e neste estudo constatou-se uma tendência dos aposentados da pesquisa a estarem vivendo conforme o planejado durante a vida laboral. As pessoas que trabalham em empresas com certa estabilidade de emprego e patrocinam planos de previdência, são favorecidas no planejamento e obtenção de um melhor bem estar após a aposentadoria. Ressalta-se que todos os participantes da pesquisa foram abordados em sua associação de aposentados, o que evidencia uma organização e preocupação com essas pessoas.

Apurou-se que os relacionamentos familiares e amigáveis ficaram mais fáceis após a aposentadoria, e este último fator é também preponderante no bem estar do aposentado. A disponibilidade de tempo foi a principal justificativa para aquelas tendências, demonstrando que quando os aposentados da pesquisa trabalhavam, tinham mais dificuldade para as questões relacionais, em função do tempo dedicado ao labor.

A mobilidade, traduzida na satisfação com o meio de transporte, pode ser explicada pelo fato da maioria dos respondentes terem carro próprio e morarem em imóvel bem localizado. Situação corroborada pela opinião dos pesquisados de que Goiânia é uma cidade atrativa para os aposentados, que apontaram vários pontos positivos, principalmente quanto à qualidade de vida e mais opções de lazer. 
Entretanto, também ficaram evidenciados os problemas de uma cidade com concentração urbana, como a violência e o trânsito tumultuado, que mereceram destaque por parte dos pesquisados, quanto aos aspectos negativos e que dificultam a vida de pessoas aposentadas nas cidades. Contudo, nesta pesquisa, confirma-se a migração para Goiânia, pois, antes de morar nesta Urbe, a maioria dos pesquisados morava em outras cidades.

Observa-se uma preocupação com a saúde, sendo este item também um dos fatores com maior significância para o bem estar. Tanto que este estudo apurou uma satisfação com a saúde depois de aposentados, e que a grande maioria dos participantes possui plano de saúde e estão fazendo mais atividades físicas e de lazer, destacando-se a prática da caminhada como a mais realizada. Os aposentados demonstraram ainda estarem mais vaidosos $\mathrm{e}$ preocupados com os cuidados físicos.

Aqui é importante ressaltar que a condição financeira não foi significativa na predição do bem estar, conforme pressuposto desta pesquisa. Entretanto, os participantes assinalaram como sugestão para os atuais trabalhadores, e futuros aposentados, que tenham uma previdência ou renda complementar como condição para o bem estar após a aposentadoria.

Diante da ausência de estudos sobre os temas aqui estudados, esta pesquisa pode ser considerada como uma primeira exploração sobre as vivências da aposentadoria. No entanto, sabemos que os resultados aqui encontrados não podem ser generalizáveis para outros tipos de participantes uma vez que nossa amostra era constituída apenas por homens de classe média e média alta. Novas pesquisas são necessárias com populações diferentes, como por exemplo, com indivíduos que recebem uma aposentadoria próxima do salário mínino ou junto aqueles que, embora aposentados, precisam continuar trabalhando para complementar a renda familiar. Finalmente, gostaríamos de ressalta que, diante do envelhecimento da população, como apontado pelo IBGE (2009), estudos sobre o período da aposentadoria vão se tornando cada vez mais prementes.

\section{REFERÊNCIAS}

American Psychiatric Association. (2002). Manual diagnóstico e estatístico dos transtornos mentais - DSM- IV - TR. (4a. Ed.). Porto Alegre: Artes Médicas.

Arias, J.L., Santin, L.J., \& Rubio, S. (2000). Effects of chronic alcohol consumption on spatial reference and working memory tasks. Alcohol, 20,149-159.
Bechara, A., Dolan, S., Denburg, N., Hindes, A., Anderson, S.W., \& Nathan, P.E. (2001). Decision-making deficits, linked to a dysfunctional ventromedial prefrontal cortex, revealed in alcohol and stimulant abusers. Neuropsychologia, 39, 376-89.

Blume, A. W., Marlatt, G. A., \& Schmaling, K. B. (2005). Memory, executive cognitive function, and readiness to change drinking behavior. Addictive Behaviors, 30(2), 301.

Calheiros, P. R. V., Oliveira, M. S., \& Andretta, I. (2006). Comorbidades Psiquiátricas no Tabagismo. Aletheia, 23, 65-74.

Carlini, E.A., Galduróz, J.C. \& Noto, L. (2001). I Levantamento Domiciliar Sobre o Uso de Drogas Psicotrópicas no Brasil-2001. São Paulo: CEBRID.

Cunha, J. (2000). Psicodiagnóstico - V. Porto Alegre: Artes Médicas.

Cunha, P.J., \& Novaes, M.A. (2004). Avaliação neurocognitiva no abuso e dependência do álcool: implicações para o tratamento. Revista Brasileira de Psiquiatria, 26(1), 23-27.

Daher, C., Berberi, R. \& Baroody, G. (2003). Effect of acute and chronic moderate alcohol comsumption on fasted and postprandial lipemia in the rat. Science Direct, 41(11), 1551-1559.

Diclemente, C C.;Schlundt, B.S.; Gemmell, L. (2004). Readiness and Stages of change in addiction treatment. American Journal on Addictions, 13, 130-119.

Fein, G., Torres, J., Price, L.J., \& Di Sclafani, V. (2006). Cognitive performance in long-term abstinent alcoholics. Alcoholiam Clinical and Experimental Research, 30(9), 1538-1544.

Figlie, N.B I; Dunn, B.; Laranjeira R. (2004). Estrutura fatorial da Stages of Change Readiness and Treatment Eagerness Scale (SOCRATES) em dependentes de álcool tratados ambulatorialmente Revista Brasileira de Psiquiatria, 26(2), 91-99.

Heaton, R. K., Chleune, G. J., Taley, J. L., Kay, G. G., \& Curtiss, G. (1993). Wisconsin card sorting test manual (Revised and expanded). Odessa: Psychological Assessment Resources.

Holmes, D. S. (1997). Psicologia dos transtornos mentais. 2. ed. Porto Alegre: Artes Médicas.

Jorge, M. R. \& Masur, J. (1986). Questionários padronizados para avaliação do grau de severidade da síndrome de dependência do álcool. Jornal Brasileiro de Psiquiatria, 35, 287-292.

Langlais, P.J., \& Ciccia, R.M. (2000). An examination of the synergistic interaction of ethanol and thiamine deficiency in the development of neurological signs and long-term cognitive and memory impairments. Alcohol Clinical Experimental Research, 25, 622-634.

McConnaughy, E. A., Prochaska, J. O., \& Velicer, W. F. (1983). Stages of change in psychoterapy: measurement and sample profiles. Psychoterapy: Theory, Research \& Practice, 20, 368-375.

Meyerhoff, D.J., Blumenfeld, R., Truran, D., Lindgren, J., Flenniken, D., Cardenas, V., Chao, L.L., Rothlind, J., Studholme, C., \& Weiner, M.W. (2004). Effects of heavy drinking, binge drinking, and family history of alcoholism 
on regional brain metabolites. Alcoholiam Clinical and Experimental Research, 28(4), 650-661.

Miller, W. \& Rollnick, S. (2001). A Entrevista Motivacional:preparando as pessoas para mudança. Porto Alegre: Artes Médicas.

Miller, W. R. \& Rollnick, S. (2001). Entrevista Motivacional: preparando as pessoas para a mudança de comportamentos adictivos. Porto Alegre: Artmed.

Mogen, B.O. (2001). Assessment of neuropsychological functions of alcoholics within an outpatient treatment program. The Sciences and Engineering, 62 (4-B),

Nassif, S. L. S. (Org.); ROSA, J. T. (Org.). Cérebro, inteligência e vínculo emocional na dependência de drogas. 1. ed. São Paulo: Vetor Editora, 2003.

Oliveira, M. S., \& Rigoni, M. S. (2005) Avaliação das funções cognitivas. In Werlang, B. S. G., Oliveira, M. S. Temas em Psicologia Clínica. Porto Alegre:EDIPUC.

Pfefferbaum, A., Sullivan, E.V., \& Rosenbloon, M.J. (2000). Pattern of motor and cognitive deficits in detoxified alcoholic men. Alcohol Clinical Experimental Research, 25, 611-621.

Prochaska, J. O., DiClemente, C. C., \& Norcross, J. C. (1992). In search of how people change: Applications to addictive behavior. American Psychologist, 47, 1102-1114.

Prochaska, J. O. \& DiClemente, C. C. (1983). Stages and processes of self-change of smoking: toward an integrative model of change. Journal of Consulting and Clinical Psychology, 51(3), 390-395.

Raistrick, D., Dunbor, G. \& Davidson, R. (1983). Development of a questionnaire to measure alcohol dependence. Britsh Journal of Addiction, 78, 89-95

Rehm, J., Room, R., Graham, K., Monteiro, M., Gmel, G. \& Sempos, C. (2003). The relationship of average volume and alcohol consumption and patterns of drinking to burden disease: an overview. Addiction, 9, 1209-1228.

Rey, A. (1959). Test de copie d'une figure complexe (Manuel). Paris: Centre de Psychologie Appliquée.

Rey, A. (1999). Teste de cópia e reprodução de memória de figuras geométricas complexas: manual. Adaptação $e$ Padronização Brasileira, (1999), $1^{\circ}$ edição; Margareth da Silva Oliveira, São Paulo: Casa do Psicólogo.

Rollnick, S., Kinnersley, P., \& Stott, N. (1993). Methods of helping patients with behaviourchange. BMJ, 307, 188190.

Velicer, W. F., Rossi, J. S., Prochaska, J. O., \& DiClemente, C. C. (1996). A criterion measurement model for health behavior change. Addictive Behaviors, 21, 555-584.

Wechsler, D. (1997). WAIS III- administration and scoring manual. San Antonio, TX: Psychological Corporation. Adaptação e Padronização Brasileira, (2004), $1^{\circ}$ edição; Elizabeth Nascimento, São Paulo: Casa do Psicólogo.

WHO World health Organization (2007). World Health Estatistic. Paris: Library Cataloguing.

Zhang, L., Welte, J. \& Wieczorek, W. (2002). The role of aggression related alcohol expectancies in explaining the link between alcohol and violent behavior. Substance Use and Misuse, 37(4), 457-471.

Zin, S., Stein, R., \& Swartzwelder, H.S. (2004). Executive Functioning Early in Abstinence From Alcohol. Alcoholiam Clinical and Experimental Research, $2 \underline{8}$ (9), 1338-1346.

Recebido em 04/09/2008 Aceito em 07/04/2009

Endereço para correspondência : Catarino de Oliveira, Programa de Pós Graduação em Ciências Ambientais e Saúde, Mestrado. Rua $232 \mathrm{n}^{\circ} 128$, área V, $3^{\circ}$ andar, Setor Universitário Goiânia, CEP 74605-140, Goiânia-GO, Brasil.E-mail: katarino@ih.com.br 
